\title{
R9 - ESTABLISHMENT OF A TANDEM CONJUGATION PROCESS AIMING IMMUNOPHENOTYPING ASSAY FOR TCD4+ LYMPHOCYTE COUNT
}

\author{
$\underline{\text { Ana Paula Araújo }}^{1}$, Patricia Barbosa Jurgilas ${ }^{1}$, Priscila Muniz da Paz ${ }^{1}$, Marta de Almeida \\ Santiago $^{2}$, Marli Sidoni ${ }^{2}$, Hilton Jorge Nascimento ${ }^{1}$, José Godinho da Silva Junior ${ }^{1}$
}

Laboratório de Macromoléculas (LAMAM) ${ }^{1}$ and Laboratório de Tecnologia Diagnóstica $(\text { LATED })^{2}$, Bio-Manguinhos, Fundação Oswaldo Cruz, Rio de Janeiro, Brasil

Objectives: The Brazillian Health Ministry demands an continuous following of lymphocytes CD4+ and CD8+ levels on HIV serum positive patients by Flow Cytometry methodology. An alternative assay has been developed by Bio-Manguinhos using four monoclonal antibodies labeled with differents fluorochromes. In this context, the aim of this work is the establishment of a conjugation process of anti-CD4 with Phycoerythrin Cyanin 7 (PECy7) fluorochrome, once this conjugate is essential to compose an immunophenotyping assay with four lymphocytes markers.

Methods: The conjugation process was based on Roederer's protocol. In the first step, Cyanin 7 (Cy7) reacted with free amino groups from Phycoerythrin (PE) to produce the Tandem fluorochrome. PECy7 was derivatizated with the crosslinking agent Succinimidyl-4-( $N$-maleimidomethyl)cyclohexane-1-carboxylate (SMCC). Meanwhile the antibody anti-CD4 was partially reduced with ditiothreitol (DTT) and further added to the activated Tandem. The conjugation reaction was stopped with N-Ethylmaleimide (NEM) to block non reacted sulphydril groups. Monoclonal antibody (anti-CD4), PE and the anti-CD4-PECy7 conjugate were evaluated by gradient-SDS-PAGE $(8-25 \%)$, IEFPAGE (3.0 - 9.0) and Size Exclusion Chromatography (SEC - Superdex 200) as process control to assure homogeneity of reactants and products. Spectrophotometric absortions at $280 \mathrm{~nm}, 565 \mathrm{~nm}, 620 \mathrm{~nm}$ and $755 \mathrm{~nm}$ were used to follow each step. At last, the antiCD4-PECy7 conjugate was analysed by flow cytometry (FC).

Results: The anti-CD4 and PE preparations were considered homogeneous by denaturing gel electrophoresis and SEC analysis. The fluorochromes PE and Cy7 presented UV-VIS spectra seemed to that described in the literature. No bleeding signal of PE was observed 
in the flow citometry analysis of anti-CD4-PECy7. Moreover, the anti-CD4-PECy7 conjugate presented similar FC results to those obtained by Becton and Dickinson's antiCD4 conjugate used as gold standard.

Conclusion: The absence of bleeding signal of PE by FC analysis suggests that all phycoeritrin emission light was absorbed by Cy7 molecules and this fact demonstrates the tandem synthesis was successful. Several check points must be performed as process control to ensure the reprodutibility of anti-CD4-PECy7 production. The behaviour of the anti-CD4-PECy7 in FC assay demonstrates that this conjugate can be used in the composition of an immunophenotyping kit for $\mathrm{TCD}^{+}$lymphocyte count as fourth marker. 